\title{
Solution Properties of Polymacromonomers Consisting of Polystyrene V. Effect of Side Chain Length on Chain Stiffness
}

\author{
Toshio HoKaJo, Ken Terao, Yo NAKamura, and Takashi NorisuYe \\ Department of Macromolecular Science, Osaka University, 1-1 Machikaneyama-cho, \\ Toyonaka, Osaka 560-0043, Japan
}

(Received February 26, 2001; Accepted April 13, 2001)

\begin{abstract}
Light scattering and viscosity measurements were made on cyclohexane and toluene solutions of a series of polymacromonomer samples consisting of polystyrene with 65 monomer units in each side chain to determine the z-average mean-square radius of gyration $\left\langle s^{2}\right\rangle_{z}$ and the intrinsic viscosity $[\eta]$ as functions of the weight average molecular weight in a range from $2.9 \times 10^{5}$ to $8.6 \times 10^{6}$. The theta temperature for the polymacromonomer in cyclohexane was determined to be $34.5^{\circ} \mathrm{C}$. The $\left\langle s^{2}\right\rangle_{2}$ data in this solvent at $34.5^{\circ} \mathrm{C}$ and toluene at $15^{\circ} \mathrm{C}$ (a good solvent) were quantitatively described by the wormlike chain with $\lambda^{-1}$ (the Kuhn segment length) $=36 \mathrm{~nm}$ and $M_{\mathrm{L}}$ (the molecular weight per unit contour length $=25000 \mathrm{~nm}^{-1}$ in the former and with $\lambda^{-1}=75 \mathrm{~nm}$ and $M_{\mathrm{L}}=25000 \mathrm{~nm}^{-1}$ in the latter. The molecular weight dependence of $[\eta]$ in each solvent was also explained by this model chain when the end effect arising from side chains near the main-chain ends was taken into account. The above $\lambda^{-1}$ values in the two solvents and the previous estimates for polystyrene polymacromonomers with shorter side chains were used to examine the dependence of the backbone stiffness on side chain length. It was found that $\lambda^{-1}$ increases almost linearly with the side-chain molecular weight for both solvents but with a larger slope in toluene than in cyclohexane.
\end{abstract}

KEY WORDS Light Scattering / Intrinsic Viscosity / Polystyrene Polymacromonomer / Wormlike Chain / Chain Stiffness /

In previous studies of this series, ${ }^{1-4}$ we found from light scattering and viscosity measurements that two polymacromonomers consisting of polystyrene with 15 and 33 styrene units in each side chain (polymacromonomers F15 and F33) behave like wormlike chains in cyclohexane at the theta temperature and in toluene, a good solvent, at $15^{\circ} \mathrm{C}$. The backbone stiffness expressed in terms of the Kuhn segment length $\lambda^{-1}$ was higher for the F33 polymer, being consistent with the earlier finding $^{5-7}$ that for polymacromonomers consisting of the poly(methyl methacrylate) backbone and polystyrene side chains in toluene (a good solvent), $\lambda^{-1}$ increases with side chain length. We also found that $\lambda^{-1}$ is larger in the good solvent than in the theta solvent for both F15 and F33. These findings ought to be theoretically explained in terms of side chain-side chain and side chainmain chain interactions, but at present, we deem it necessary to extend the experimental work to a polystyrene polymacromonomer with a larger side chain length in order to establish the experimental relations between the backbone stiffness and the side chain length in both theta and good solvents.

Thus we prepared polystyrene polymacromonomer samples with 65 side chain units and different mainchain lengths, and determined $\mathrm{z}$-average mean-square radii of gyration $\left\langle s^{2}\right\rangle_{z}$ and intrinsic viscosities [ $\eta$ ] for them by static light scattering and viscometry in cyclohexane at the theta temperature and in toluene at $15^{\circ} \mathrm{C}$. In the work reported below, we analyze the results on the basis of the wormlike chain and compare the estimated parameters with those for the F15 and F33 polymacromonomers.

\section{EXPERIMENTAL}

\section{Preparation of Polymacromonomer Samples}

Styrene was anionically polymerized in toluene using tolyllithium as an initiator, and the reaction was terminated by $p$-chloromethylstyrene (Seimi Chemical Co. Ltd.) to obtain the macromonomer. ${ }^{8}$ Before the termination, an aliquot of the living polystyrene solution was taken out, and linear polystyrene was precipitated by the addition of methanol. Its weight-average molecular weight $M_{\mathrm{w}}$ was 6770 when determined by light scattering in toluene. The crude macromonomer was reprecipitated several times into methanol to remove the excess $p$-chloromethylstyrene. The complete removal of this impurity was confirmed by ${ }^{1} \mathrm{H} \mathrm{NMR}$, which showed the end group functionality of this macromonomer to be about $75 \%$. The macromonomer thus purified was polymerized in benzene at $38-60^{\circ} \mathrm{C}$ with azobisisobutylonitrile as an initiator. The monomer concentration was adjusted to 84 $-87 \%$ by weight. The yield of the polymacromonomer changed from 56 to $73 \%$ depending on monomer concentration and temperature.

The polymacromonomer samples prepared were fractionated by repeated fractional precipitation as described previously. ${ }^{1}$ Seven fractions (designated below as F65-1, F65-2, ... , F65-7) with different main-chain lengths were chosen for the present work. Weight- to number-average molecular weight ratios were estimated to be 1.09 for F65-3 and F65-4, 1.10 for F65-2, 1.13 for $F$ 65-5, and 1.27 for F65-6 by gel permeation chromatography with the $M_{\mathrm{w}}$ - elution volume relation established for this polymacromonomer. 


\section{Light Scattering}

Light scattering measurements were made on a Fica50 light-scattering photometer using vertically polarized incident light of 436 and $546 \mathrm{~nm}$ in wavelength. The instrument was calibrated with benzene at $25^{\circ} \mathrm{C}$ and the literature values for the Rayleigh ratio. ${ }^{9}$ Each polymacromonomer solution was made optically clean by centrifugation at $12000 \mathrm{rpm}$ for $1.5 \mathrm{~h}$, carefully sucked with a clean pipette, and transferred into a cylindrical light scattering cell. Intensity data were taken at 15 and $34.5^{\circ} \mathrm{C}$ for toluene and cyclohexane solutions, respectively; for samples F65-7 and F65-4 in cyclohexane, data were also taken at other temperatures $T$ of $25,30,40$, and $45^{\circ} \mathrm{C}$.

The polymer mass concentration $c$ was calculated from the weight fraction with the solution density $\rho$, which was determined as a function of $T$ and $c$ for F65-4 using an Anton-Paar DMA 5000 density meter. The results were found to be represented by

$$
\begin{aligned}
\rho= & \left.0.87149+0.199 c \quad \text { (in toluene at } 15^{\circ} \mathrm{C}\right) \\
\rho= & 0.76481-0.00072\left(T /{ }^{\circ} \mathrm{C}-34.5\right)+[0.282 \\
& \left.+0.0004\left(T /{ }^{\circ} \mathrm{C}-34.5\right)\right] c
\end{aligned}
$$

(in cyclohexane)

in units of $\mathrm{g} \mathrm{cm}^{-3}$. Specific refractive index increments $\partial n / \partial c$ for $\mathrm{F} 65-7$ in cyclohexane at $34.5^{\circ} \mathrm{C}$ and toluene at $15^{\circ} \mathrm{C}$ were measured using a modified Schulz-Cantow type differential refractometer. The results were 0.107 $\mathrm{cm}^{3} \mathrm{~g}^{-1}$ at $436 \mathrm{~nm}$ and $0.104 \mathrm{~cm}^{3} \mathrm{~g}^{-1}$ at $546 \mathrm{~nm}$ in toluene and $0.180 \mathrm{~cm}^{3} \mathrm{~g}^{-1}$ at $436 \mathrm{~nm}$ and $0.171 \mathrm{~cm}^{3} \mathrm{~g}^{-1}$ at $546 \mathrm{~nm}$ in cyclohexane.

\section{Viscometry}

Viscosity measurements were made using Ubbelohde type viscometers. The relative viscosity was evaluated by taking account of the difference between the solution and solvent densities. The Huggins plot, ${ }^{10}$ the Fuoss ${ }^{-}$ Mead plot, ${ }^{11}$ and the Billmeyer plot $^{12}$ were combined to determine $[\eta]$ and the Huggins constant $k^{\prime}$.

\section{RESULTS}

Figure 1 shows the angular dependence of $\left(K c / R_{\theta}\right)^{1 / 2}$ for sample $\mathrm{F} 65-7$ in cyclohexane at $34.5^{\circ} \mathrm{C}$. Here, $K$ is the optical constant and $R_{\theta}$ is the excess reduced scattering intensity at scattering angle $\theta$. Extrapolation of the data points at the respective concentrations gives almost identical intercepts, i.e., equal zero-angle values of $K c / R_{\theta}$ within experimental error.
The second virial coefficients $A_{2}$ obtained for sample F65-7 as a function of $T$ are shown in Figure 2; the $A_{2}$ values at the temperatures other than $34.5^{\circ} \mathrm{C}$ have been evaluated from $A_{2} M_{\mathrm{w}}$ with the $M_{\mathrm{w}}$ value at $34.5^{\circ} \mathrm{C}$. It can be seen that $A_{2}$ vanishes at $34.5^{\circ} \mathrm{C}$, the theta temperature for linear polystyrene and polymacromonomers F15 and F33 in cyclohexane. ${ }^{1,2}$ For another sample F65-4, the absolute values of $A_{2}$ at $30-45^{\circ} \mathrm{C}$ were less than $8 \times$ $10^{-6} \mathrm{~cm}^{3} \mathrm{~mol} \mathrm{~g}{ }^{-2}$, being hardly distinguishable from zero. We therefore concluded that the theta temperature of the F65 polymacromonomer in cyclohexane is practically the same as that $\left(34.5^{\circ} \mathrm{C}\right)$ of $\mathrm{F} 15$ and F33. In fact, the $A_{2}$ values for all our samples in the solvent were essentially zero at this temperature. The numerical results of $M_{\mathrm{w}},\left\langle s^{2}\right\rangle_{z}$, and $A_{2}$ in cyclohexane and toluene are presented in Tables I and II, respectively.

Figure 3 shows the molecular weight dependence of $\left\langle s^{2}\right\rangle_{z}$ for the $\mathrm{F} 65$ polymacromonomer in toluene $\left(15^{\circ} \mathrm{C}\right)$

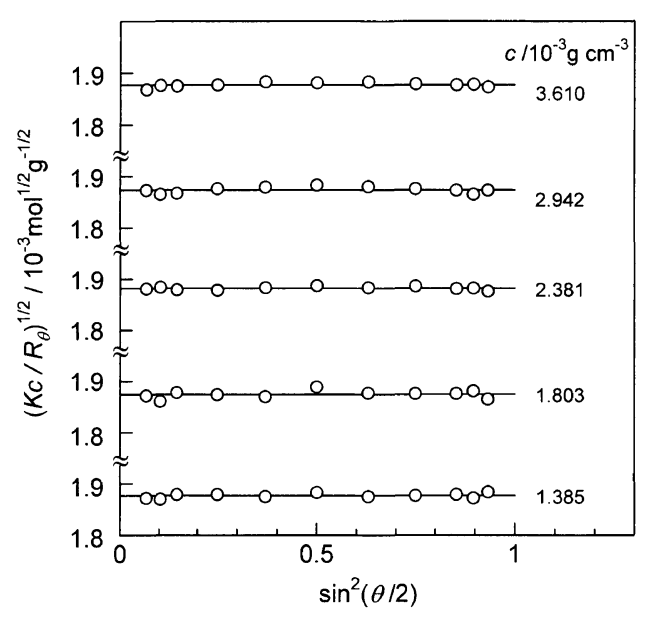

Figure 1. Angular dependence of $\left(K c / R_{\theta}\right)^{1 / 2}$ at indicated $c$ for polymacromonomer sample $\mathrm{F} 65-7$ in cyclohexane at $34.5^{\circ} \mathrm{C}$.

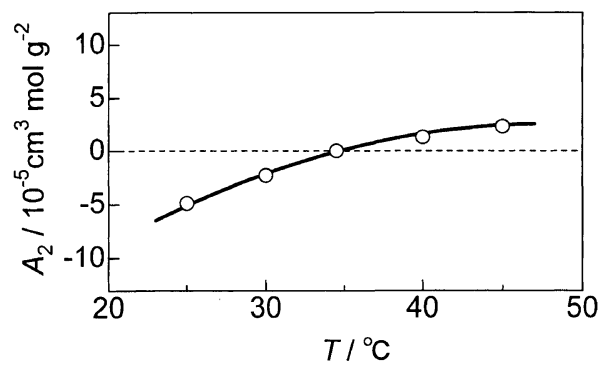

Figure 2. Temperature dependence of $A_{2}$ for sample F65-7 in cyclohexane.

Table I. Results from light scattering and viscosity measurements on polymacromonomer samples of $\mathrm{F} 65$ in cyclohexane at $34.5^{\circ} \mathrm{C}$

\begin{tabular}{ccccc}
\hline Sample & $M_{\mathrm{w}} / 10^{5}$ & $\left\langle\mathrm{~s}^{2}\right\rangle_{\mathrm{z}} / \mathrm{nm}^{2}$ & {$[\eta] / \mathrm{cm}^{3} \mathrm{~g}^{-1}$} & $k^{\prime}$ \\
\hline F65-1 & 85.5 & 1670 & 31.4 & 0.76 \\
F65-2 & 42.3 & 790 & 19.3 & 0.83 \\
F65-3 & 36.4 & 641 & 14.8 & 0.87 \\
F65-4 & 20.9 & 287 & 11.9 & 0.91 \\
F65-5 & 14.5 & 165 & 9.9 & 0.93 \\
F65-6 & $(9.43)^{\mathrm{a}}$ & - & 8.4 & 0.97 \\
\hline
\end{tabular}

\footnotetext{
${ }^{a}$ In toluene.
} 
Table II. Results from light scattering and viscosity measurements on polymacromonomer samples of F65 in toluene at $15^{\circ} \mathrm{C}$

\begin{tabular}{|c|c|c|c|c|c|}
\hline Sample & $M_{\mathrm{w}} / 10^{5}$ & $\left\langle s^{2}\right\rangle_{\mathrm{z}} / \mathrm{nm}^{2}$ & $10^{5} A_{2} / \mathrm{cm}^{3} \mathrm{~mol} \mathrm{~g}^{-2}$ & {$[\eta] / \mathrm{cm}^{3} \mathrm{~g}^{-1}$} & $k^{\prime}$ \\
\hline F65-1 & 81.6 & 2970 & 1.6 & 64.2 & 0.45 \\
\hline F65-2 & 40.6 & 1270 & 1.3 & - & - \\
\hline F65-3 & 36.6 & 902 & 1.4 & 31.9 & 0.45 \\
\hline F65-4 & 21.6 & 394 & 1.8 & 21.9 & 0.54 \\
\hline F65-5 & 14.7 & 218 & 2.6 & 16.7 & 0.65 \\
\hline F65-6 & 9.43 & - & 2.4 & 13.9 & 0.75 \\
\hline F65-7 & $(2.86)^{\mathrm{a}}$ & - & - & 11.1 & 0.93 \\
\hline
\end{tabular}

${ }^{\mathrm{a}}$ In cyclohexane.

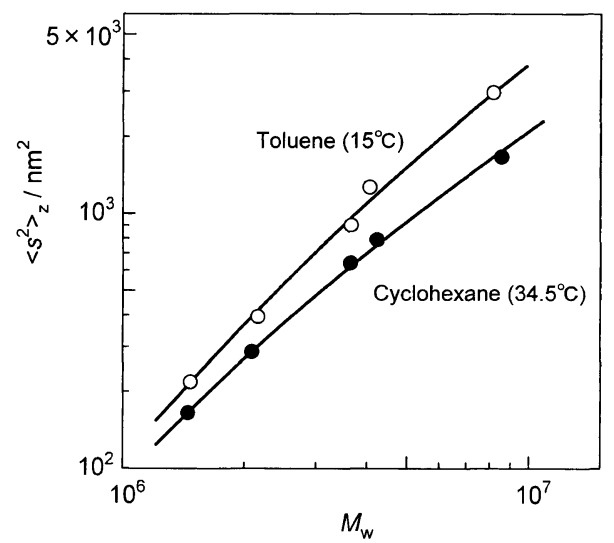

Figure 3. Molecular weight dependence of $\left\langle s^{2}\right\rangle_{z}$ for the F65 polymacromonomer in toluene $15^{\circ} \mathrm{C}$ (unfilled circles) and in cyclohexane $34.5^{\circ} \mathrm{C}$ (filled circles). The solid lines represent the theoretical values calculated from eq 1 with the parameters in Table III.

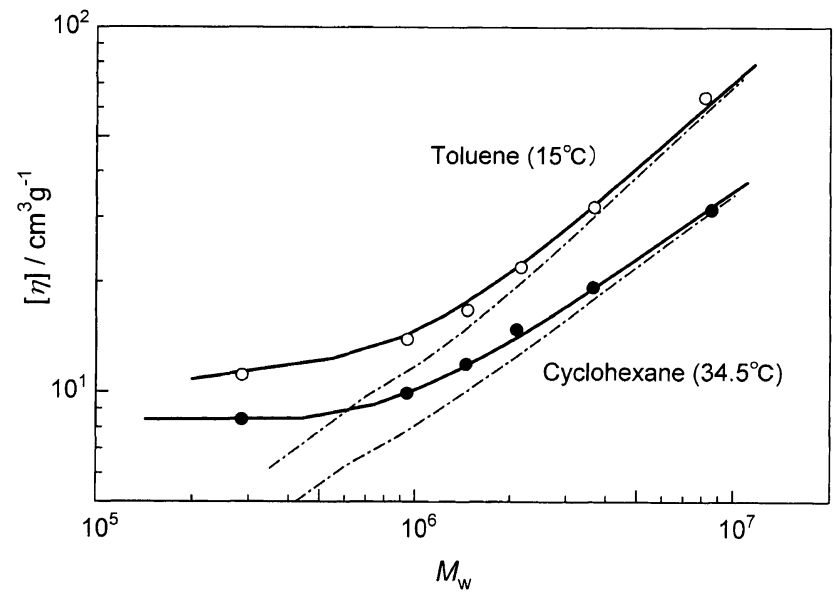

Figure 4. Molecular weight dependence of $[\eta]$ for the F65 polymacromonomer in the indicated solvents. The symbols are the same as those used in Figure 3. Solid lines, theoretical values calculated from eq 3 with the parameters in Table III; dot-dashed lines, theoretical values for $\delta=0$.

and cyclohexane $\left(34.5^{\circ} \mathrm{C}\right)$. The curves for cyclohexane and toluene solutions have slopes 1.4 and 1.6 , respectively, for $M_{\mathrm{w}}<3 \times 10^{6}$ and bend down with increasing $M_{\mathrm{w}}$. Such behavior of $\left\langle s^{2}\right\rangle_{\mathrm{z}}$ indicates that our polymacromonomer is semiflexible in both solvents.

The numerical values of $[\eta]$ in cyclohexane and toluene are included in Tables I and II, along with those of $k^{\prime}$, and the molecular weight dependence of $[\eta]$ in the two solvents is shown in Figure 4. The solid lines closely fitting the data points in the respective solvents rise very gradually up to $M_{\mathrm{w}} \sim 10^{6}$ and bend up relatively

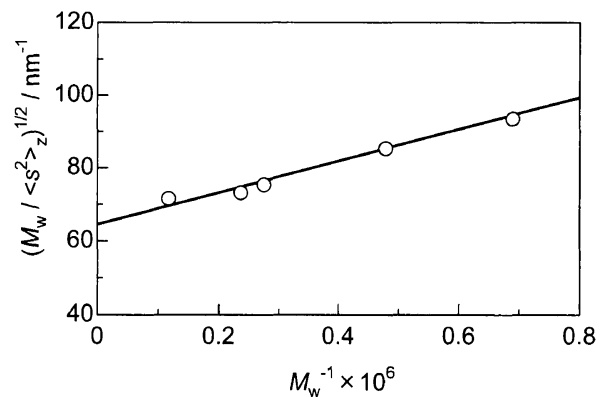

Figure 5. Plot of $\left(M_{\mathrm{w}} /\left\langle s^{2}\right\rangle_{\mathrm{z}}\right)^{1 / 2}$ vs. ${M_{\mathrm{w}}}^{-1}$ for F65 samples in cyclohexane at $34.5^{\circ} \mathrm{C}$.

sharply with further increasing $M_{\mathrm{w}}$. Their slopes reach 0.85 and 0.55 for toluene and cyclohexane solutions, respectively. The very weak molecular weight dependence of $[\eta]$ for low $M_{\mathrm{w}}$ is typical of polymacromonomers. ${ }^{3,5,13}$ The significance of the indicated solid and dashed lines is interpreted in the next section.

\section{DISCUSSION}

\section{Mean-Square Radius of Gyration}

The mean-square radius of gyration of the unperturbed wormlike chain with a contour length $L$ and a Kuhn segment length $\lambda^{-1}$ is expressed as ${ }^{14}$

$$
\left\langle s^{2}\right\rangle=\frac{L}{6 \lambda}-\frac{1}{4 \lambda^{2}}+\frac{1}{4 \lambda^{3} L}-\frac{1}{8 \lambda^{4} L^{2}}[1-\exp (-2 \lambda L)]
$$

When $\lambda L>2$, this equation may be approximated by ${ }^{15}$

$$
\left(M /\left\langle s^{2}\right\rangle\right)^{1 / 2}=\left(6 \lambda M_{\mathrm{L}}\right)^{1 / 2}\left(1+3 M_{\mathrm{L}} / 4 \lambda M\right)
$$

where $M$ is the molecular weight and $M_{\mathrm{L}}$ is the molar mass per unit contour length of the chain, i.e., $M_{\mathrm{L}}=$ $M / L$. Equation 2 indicates that $\lambda^{-1}$ and $M_{\mathrm{L}}$ can be determined from the intercept and slope of a plot of $(M /$ $\left.\left\langle s^{2}\right\rangle\right)^{1 / 2}$ vs. $M^{-1}$.

Figure 5 shows the plot of $\left(M_{\mathrm{w}} /\left\langle s^{2}\right\rangle_{\mathrm{z}}\right)^{1 / 2} v s . M_{\mathrm{w}}^{-1}$ constructed from the present $\left\langle s^{2}\right\rangle_{\mathrm{z}}$ data in cyclohexane at the theta temperature. The data points follow a straight line, which yields $\lambda^{-1}=36 \mathrm{~nm}$ and $M_{\mathrm{L}}=25000 \mathrm{~nm}^{-1}$. This $M_{\mathrm{L}}$ is much larger than the previously determined values $\left(6200\right.$ and $13000 \mathrm{~nm}^{-1}$ ) for the polymacromonomers F15 and F33 in the same solvent, ${ }^{1-4}$ reflecting the longer side chains of our polymer. Importantly, however, it yields $0.27 \mathrm{~nm}$ for the contour length per main chain residue, a value close to those obtained previously for these polymacromonomers ${ }^{1,2}$ and also that calculated for 
linear polystyrene with the all-trans conformation.

The solid line for cyclohexane solutions in Figure 3 actually represents the theoretical values calculated form eq 1 with the above parameters. Its satisfactory fit to the filled circles suggests that the effects of chain thickness and chain ends on $\left\langle s^{2}\right\rangle$ are insignificant in the molecular weight range examined. To confirm this, we estimated the contribution from side chains to $\left\langle s^{2}\right\rangle$ using the wormlike comb model, in which wormlike side chains with stiffness $\lambda_{\mathrm{s}}{ }^{-1}$ and contour length $L_{\mathrm{s}}$ are linked to the main chain by completely flexible joints. The equation for $\left\langle s^{2}\right\rangle$ of this model chain can be found in ref 16 (eqs. 4-6). Using the molecular weight of the side chain along with $M_{\mathrm{L}}=390 \mathrm{~nm}^{-1}$ and $\lambda_{\mathrm{s}}^{-1}=2 \mathrm{~nm}$ for linear polystyrene, ${ }^{17}$ we find that the contribution from side chains to $\left\langle s^{2}\right\rangle^{1 / 2}$ is only about $4.5 \%$ for the lowest molecular weight sample (F65-5) examined and becomes negligible with increasing $M_{\mathrm{w}}$. Hence, it introduces no substantial error in the analysis of the present $\left\langle s^{2}\right\rangle_{z}$ data.

In analyzing the toluene data, we employed the curvefitting procedure, because we anticipated that the condition $\lambda L>2$ for eq 2 is not satisfied for the two lowest molecular weights. The curve for toluene solutions in Figure 3 shows the best fit obtained with $M_{\mathrm{L}}=25000 \mathrm{~nm}^{-1}$ and $\lambda^{-1}=75 \mathrm{~nm}$. This $M_{\mathrm{L}}$ value agrees with that in cyclohexane estimated above. On the other hand, the $\lambda^{-1}$ value of $75 \mathrm{~nm}$ in toluene is more than twice as large as that $(36 \mathrm{~nm})$ in cyclohexane, indicating that the polymacromonomer backbone is much stiffer in the good solvent than in the theta solvent. The following remark may be pertinent here with regard to the analysis of the toluene data made without consideration of intramolecular excluded-volume effect.

For the polystyrene polymacromonomers F15 and F33 in toluene, excluded-volume effects on $\left\langle s^{2}\right\rangle_{z}$ became appreciable when $\lambda L$ exceeded $5-6$. This critical $\lambda L$ for the onset of volume effect is comparable to those for linear flexible chains in good solvents, but it is almost one order of magnitude smaller than those for typical stiff chains ${ }^{18}$ probably because of the much higher thickness of the polymacromonomer chains. In other words, though polymacromonomers behave like stiff chains, their excluded-volume effects begin to appear at a small $\lambda L$ of about 5 as in linear flexible chains. For our polymacromonomer, this $\lambda L$ corresponds to a molecular weight of $9.4 \times 10^{6}$, which is slightly larger than the highest $M_{\mathrm{w}}$ studied here. Thus, the above analysis of the toluene data may be rationalized.

\section{Intrinsic Viscosity}

A polymacromonomer molecule in solution may be modeled by a cylindrical wormlike chain if its side chains are included as the continuous body, but theoreti- cal values of $[\eta]$ for the wormlike cylinder model ${ }^{19}$ are unavailable for large cylinder diameters $d$ relative to $\lambda^{-1}, i . e .$, for $\lambda d>0.2$. In our previous work, Terao et al. ${ }^{3}$ examining the limited information on the cylinder model (capped with hemispheres at both ends) and the theory for the touched-bead model, ${ }^{20}$ reached the conclusion that the theoretical $[\eta]$ for the former model with $\lambda d>$ 0.2 can be replaced in a good approximation by that for the latter model with the bead diameter $d_{\mathrm{b}}$ equal to $d$. This allows the desired $[\eta]$ for wormlike cylinders with $\lambda d>0.2$ to be expressed as

$$
[\eta]=f(\lambda L, \lambda d) / \lambda^{3} M
$$

where $f(\lambda L, \lambda d)$ is a known function ${ }^{20}$ of $\lambda L$ and $\lambda d(=$ $\left.\lambda d_{\mathrm{b}}\right)$. Though $[\eta]$ in this equation is given for the discrete number of beads, we regard it as a continuous function of $L$.

Terao et $a l .{ }^{3}$ further considered that some side chains near the main-chain ends of a polymacromonomer molecule contribute toward apparently increasing the main chain length (see Figure 3 of ref 3 ), and redefined $L$ by

$$
L=M / M_{\mathrm{L}}+\delta
$$

where $\delta$ stands for the contribution from such side chains to the main-chain contour length $(\delta / 2$ from each end). Consideration of the end effect (i.e., the effect of $\delta$ ) was essential to explain the weak $M_{\mathrm{w}}$-dependence of $[\eta]$ observed for the polymacromonomers F15 and F33 in cyclohexane and toluene.

Equation 3 with eq 4 contains $M_{\mathrm{L}}, \lambda^{-1}, d$, and $\delta$ as the parameters, but all of them cannot uniquely be determined from our $[\eta]$ data. In the present analysis, we assumed the $\lambda^{-1}$ values from the above analysis of $\left\langle s^{2}\right\rangle_{\mathrm{z}}$, i.e., $\lambda^{-1}=36 \mathrm{~nm}$ in cyclohexane and $\lambda^{-1}=75 \mathrm{~nm}$ in toluene, and estimated the other parameters in each solvent by curve fitting. The solid lines for the respective solvents in Figure 4 represents the resulting best-fit theoretical values of $[\eta]$. The agreements between the measured and calculated $[\eta]$ in both solvents are good. A few breaks of each curve in the low molecular weight region are due to the fact that the theoretical $[\eta]$ values for discrete bead numbers are connected by straight lines. The parameters used for the calculation are presented in Table III, along with those determined from $\left\langle s^{2}\right\rangle_{\mathrm{z}}$. The values of $M_{\mathrm{L}}$ from $\left\langle s^{2}\right\rangle_{\mathrm{z}}$ and $[\eta]$ in either solvent agree almost perfectly, indicating that our analyses consistently explain the behavior of the two properties.

The dot-dashed lines in Figure 4 refer to the theoretical $[\eta]$ values for $\delta=0$. Their downward deviations from the data points in the respective solvents are remarkable even at a molecular weight as high as $10^{6}$, demonstrating the substantial end effects on $[\eta]$. We note that,

Table III. Model parameters for polymacromonomer F65 in cyclohexane and toluene

\begin{tabular}{cccccc}
\hline Solvent & Data & $\lambda^{-1} / \mathrm{nm}$ & $10^{-3} M_{\mathrm{L}} / \mathrm{nm}^{-1}$ & $d / \mathrm{nm}$ & $\delta / \mathrm{nm}$ \\
\hline $\mathrm{CH}^{\mathrm{a}}$ & $\left\langle s^{2}\right\rangle_{\mathrm{z}}$ & 36 & 25.0 & - & - \\
$\mathrm{CH}$ & {$[\eta]^{\mathrm{c}}$} & $36^{\mathrm{c}}$ & 26.0 & 11.5 & 6 \\
Tol $^{\mathrm{b}}$ & $\left\langle s^{2}\right\rangle_{z}$ & 75 & 25.0 & - & - \\
Tol & {$[\eta]^{\mathrm{c}}$} & $75^{\mathrm{c}}$ & 25.0 & 14 & 6 \\
\hline
\end{tabular}

\footnotetext{
${ }^{a}$ Cyclohexane at $34.5^{\circ} \mathrm{C} .{ }^{\mathrm{b}}$ Toluene at $15.0{ }^{\circ} \mathrm{C} .{ }^{\mathrm{c}}$ From $\left\langle s^{2}\right\rangle_{\mathrm{z}}$.
} 


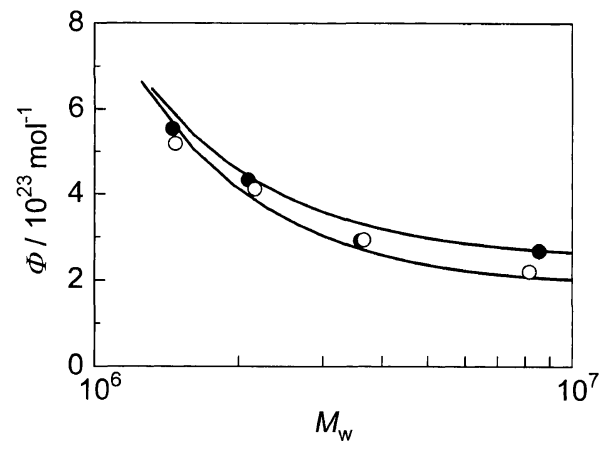

Figure 6. Experimental $\Phi$ for the F65 polymacromonomer compared with the theoretical curves. Symbols are the same as those used in Figure 3.

as expected, the value of $\delta$ for our polymacromonomer (Table III) is considerably larger than those $(2.2-4.3$ nm) for F15 and F33 with shorter side chains.

In the previous study ${ }^{3}$ on F15 and F33, we found that the $d$ value determined from $[\eta]$ for each polymer in cyclohexane is almost twice as large as the unperturbed root-mean-square end-to-end distance $\left\langle r^{2}\right\rangle^{1 / 2}$ of the linear polystyrene chain with the same contour length as that of each side chain in the polymacromonomer. To examine whether this is also the case for our F65 polymacromonomer, we calculated $\left\langle r^{2}\right\rangle^{1 / 2}$ for linear polystyrene with the molecular weight of each side chain of F65 using the wormlike chain model with $M_{\mathrm{L}}=390 \mathrm{~nm}^{-1}$ and $\lambda^{-1}=2 \mathrm{~nm} .{ }^{17}$ The result gave $\left\langle r^{2}\right\rangle^{1 / 2}=5.7 \mathrm{~nm}$, which is very close to a half the $d$ value determined from $[\eta]$ in cyclohexane. We note that essentially the same $\left\langle r^{2}\right\rangle^{1 / 2}$ value is obtained when the helical wormlike chain with the known parameter set ${ }^{19}$ is used for the polystyrene side chain.

Figure 6 shows the Flory viscosity factor $\Phi[=[\eta] M /(6$ $\left.\left\langle s^{2}\right\rangle\right)^{3 / 2}$ ] plotted against $\log M_{\mathrm{w}}$. It can be seen that $\Phi$ 's for both cyclohexane and toluene systems decrease sharply with increasing molecular weight. Similar behavior of $\Phi$ was previously observed for F15 and F33, ${ }^{3}$ but a point to note is that the decrease in $\Phi$ with an increase in $M_{\mathrm{w}}$ becomes less pronounced with decreasing side chain length. The solid lines in the figure represent the theoretical values calculated from eqs 1,3 , and 4 with $\lambda^{-1}=36 \mathrm{~nm}, M_{\mathrm{L}}=25500 \mathrm{~nm}^{-1}$ (the mean of the values from $\left\langle s^{2}\right\rangle_{\mathrm{z}}$ and $\left.[\eta]\right), d=11.5 \mathrm{~nm}$, and $\delta=6 \mathrm{~nm}$ for cyclohexane solutions and $\lambda^{-1}=75 \mathrm{~nm}, M_{\mathrm{L}}=25000$ $\mathrm{nm}^{-1}, d=14 \mathrm{~nm}$, and $\delta=6 \mathrm{~nm}$ for toluene solutions (note that $\delta=0$ for $\left\langle s^{2}\right\rangle$ ). The agreement between the experimental and theoretical values are satisfactory for both solvents, confirming that the cylindrical wormlike chain is a good model for $[\eta]$ of our polymacromonomer.

\section{Dependence of Backbone Stiffness on Side Chain Length}

The values of $\lambda^{-1}$ estimated form $\left\langle s^{2}\right\rangle_{\mathrm{z}}$ for our polymacromonomers in cyclohexane and toluene are much larger than those for F15 and F33 in the corresponding solvents (see Table 4 of ref 2 ), ascertaining that $\lambda^{-1}$ for the polystyrene polymacromonomers in either solvent is an increasing function of side chain length.

Figure 7 shows how $\lambda^{-1}$ increases with increasing the side chain molecular weight $M_{\text {side }}$. The plotted points approximately follow straight lines for both solvents but

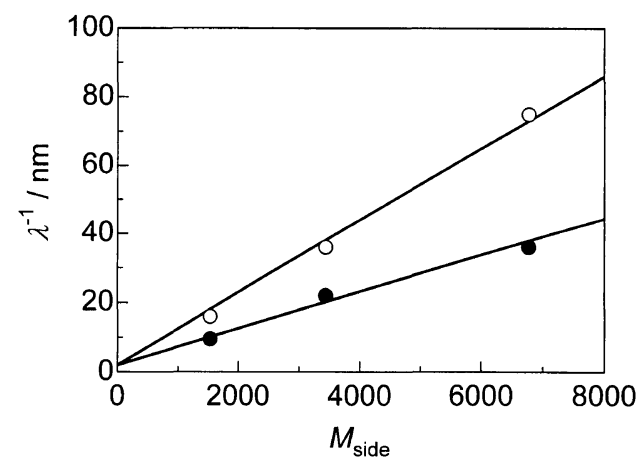

Figure 7. Dependence of $\lambda^{-1}$ on the side chain molecular weight of polystyrene polymacromonomer. Lines are eye guides.

with a larger slope in toluene than in cyclohexane. The increase in $\lambda^{-1}$ with $M_{\text {side }}$ for toluene solutions suggests that binary excluded-volume interactions between side chains stiffen the polymacromonomers. However, this idea does not explain the stiffness of the polymacromonomers in cyclohexane, because effective binary interactions between segments are known to vanish at the theta point. $^{21}$ The stiffness in the theta solvent may be considered to arise primarily from ternary interactions among side chain segments. This will be discussed in a forthcoming paper on some theoretical basis.

\section{REFERENCES}

1. K. Terao, Y. Takeo, M. Tazaki, Y. Nakamura, and T. Norisuye, Polym. J., 31, 193 (1999).

2. K. Terao, Y. Nakamura, and T. Norisuye, Macromolecules, 32, 711 (1999).

3. K. Terao, T. Hokajo, Y. Nakamura, and T. Norisuye, Macromolecules, 32, 3690 (1999).

4. K. Terao, S. Hayashi, Y. Nakamura, and T. Norisuye, Polym. Bull., 44, 309 (2000).

5. M. Wintermantel, M. Schmidt, Y. Tsukahara, K. Kajiwara, and S. Kohjiya, Macromol. Rapid Commun., 15, 279 (1994).

6. N. Nemoto, M. Nagai, A. Koike, and S. Okada, Macromolecules, 28, 3854 (1995).

7. M. Wintermantel, M. Gerle, K. Fischer, M. Schmidt, I. Wataoka, H. Urakawa, K. Kajiwara, and Y. Tsukahara, Mac romolecules, 29, 978 (1996).

8. Y. Tsukahara, J. Inoue, Y. Ohta, S. Kohjiya, and Y. Okamoto, Polym. J., 26, 1013 (1994).

9. Gj Deželić and J. Vavra, Croat. Chem. Acta, 38, 35 (1966).

10. M. L. Huggins, J. Am. Chem. Soc., 64, 2716 (1942).

11. D. F. Mead and R. M. Fuoss, J. Am. Chem. Soc., 64, 277 (1942).

12. F. W. Billmeyer, Jr., J. Polym. Sci., 4, 83 (1949).

13. Y. Tsukahara, S. Kohjiya, K. Tsutsumi, and Y. Okamoto, Macromolecules, 27, 1662 (1994).

14. H. Benoit and P. Doty, J. Phys. Chem., 57, 958 (1953).

15. H. Murakami, T. Norisuye, and H. Fujita, Macromolecules, 13, $345(1980)$.

16. Y. Nakamura, Y. Wan, J. W. Mays, H. Iatrou, and N. Hadjichristidis, Macromolecules, 33, 8323 (2000); correction for eq 6 appears in Macromolecules, 34, 2018 (2001).

17. T. Norisuye and H. Fujita, Polym. J., 14, 143 (1982).

18. T. Norisuye, Prog. Polym. Sci., 18, 543 (1993).

19. H. Yamakawa, "Helical Wormlike Chains in Polymer Solutions," Springer-Verlag GmbH \& Co., Berlin, 1997.

20. T. Yoshizaki, I. Nitta, and H. Yamakawa, Macromolecules, 21, 165 (1988).

21. Y. Nakamura, T. Norisuye, and A. Teramoto, Macromolecules, 24, 4904 (1991). 